\title{
A Principal Component Regression Approach for Estimating Ventricular Repolarization Duration Variability
}

\author{
Mika P. Tarvainen, ${ }^{1}$ Tomi Laitinen, ${ }^{2}$ Tiina Lyyra-Laitinen, ${ }^{2}$ Juha-Pekka Niskanen, ${ }^{1}$ and Pasi A. Karjalainen ${ }^{1}$ \\ ${ }^{1}$ Department of Physics, University of Kuopio, P.O. Box 1627, 70211 Kuopio, Finland \\ ${ }^{2}$ Department of Clinical Physiology and Nuclear Medicine, Kuopio University Hospital, P.O. Box 1777, 70211 Kuopio, Finland
}

Received 28 April 2006; Revised 27 September 2006; Accepted 29 October 2006

Recommended by Pablo Laguna Lasaosa

Ventricular repolarization duration (VRD) is affected by heart rate and autonomic control, and thus VRD varies in time in a similar way as heart rate. VRD variability is commonly assessed by determining the time differences between successive R- and T-waves, that is, RT intervals. Traditional methods for RT interval detection necessitate the detection of either T-wave apexes or offsets. In this paper, we propose a principal-component-regression- (PCR-) based method for estimating RT variability. The main benefit of the method is that it does not necessitate T-wave detection. The proposed method is compared with traditional RT interval measures, and as a result, it is observed to estimate RT variability accurately and to be less sensitive to noise than the traditional methods. As a specific application, the method is applied to exercise electrocardiogram (ECG) recordings.

Copyright (c) 2007 Mika P. Tarvainen et al. This is an open access article distributed under the Creative Commons Attribution License, which permits unrestricted use, distribution, and reproduction in any medium, provided the original work is properly cited.

\section{INTRODUCTION}

Ventricular repolarization duration (VRD) is known to be affected by heart rate (HR) and autonomic control (mainly through sympathetic branch), and thus VRD varies in time in a similar way as HR $[1,2]$. The time interval between Qwave onset and T-wave offset in an electrocardiogram (ECG), that is, QT interval, corresponds to the total ventricular activity including both depolarization and repolarization times, and thus QT interval may be used as an index of VRD. It has been suggested that abnormal QT variability could be a marker for a group of severe cardiac diseases such as ventricular arrhythmias [3]. In addition, it has been suggested that QT variability could yield such additional information which cannot be observed from HR variability [4].

Due to the difficulty in fixing automatically the Q-wave onset in VRD determination, RT interval is typically used instead of QT interval $[5,6]$. The RT interval can be defined as the interval from $\mathrm{R}$-wave apex either to $\mathrm{T}$-wave apex $\left(\mathrm{RT}_{\text {apex }}\right)$ or to $\mathrm{T}$-wave offset $\left(\mathrm{RT}_{\text {end }}\right)$. The T-wave apex is typically fixed by fitting a parabola around the T-wave maximum [5]. The T-wave offset, on the other hand, can be fixed with a number of methods. In threshold methods, the T-wave offset is fixed as an intercept of the T-wave or its derivative with a threshold level above the isoelectric line [7-9]. In the fitting methods, the T-wave offset is fixed, for example, as an intercept of a line fitted to T-wave downslope with the isoelectric line $[8,10]$.

The automatic RT interval measures have been compared with manual measurements, for example, in $[11,12]$. In addition, different automatic methods for RT interval estimation have been compared, for example, in $[8,9,13]$. Even though the selection of the optimal RT interval measure was found to depend on the type of the simulated noise, in most of the cases, $\mathrm{RT}_{\text {apex }}$ measure gave the most accurate results. The $\mathrm{RT}_{\text {apex }}$ measure is also relatively easy to implement, and thus it has been sometimes preferred to $\mathrm{RT}_{\text {end }}$ measures, although the variability of the T-wave downslope has been found to hide important physiological information $[10,14]$.

In this paper, we propose a robust method for estimating the variation in the RT interval. The method is based on principal component regression (PCR) and it does not necessitate T-wave detection. In the method, T-wave epochs are extracted from ECG in respect of R-wave fiducial points and the variability in the RT interval is reflected on the principal components of the epoch data. It should be noted that the proposed method does not give absolute values for RT interval, but estimates the variation in the RT interval. The variability estimates obtained with the method are compared with traditional $\mathrm{RT}_{\text {apex }}$ and $\mathrm{RT}_{\text {end }}$ measures. The noise sensitivity of the proposed method is evaluated by examining the 
effect of simulated Gaussian noise on the spectral characteristics of the estimated RT variability series. As a specific application, the proposed method is finally applied to exercise ECG and the interrelationships between RR and RT intervals variability are considered.

\section{MATERIALS AND METHODS}

The estimation of RT interval is not always a simple task. Twave is a smooth waveform that can be hard to detect accurately in conditions where the signal-to-noise ratio (SNR) is not high enough. Several artifacts also affect the reliability of the detection remarkably. In this section, we first describe the performed ECG measurements and the three traditional RT interval measurement methods which are used here as reference methods. After that, the PCR-based method for estimating RT interval variability and the approach for evaluating the noise sensitivity of different RT measures are described.

\subsection{ECG measurements}

The ECG measurements utilized in this paper consist of a single resting ECG measurement and five exercise ECG measurements. In all measurements, ECG electrodes were placed according to the conventional 12-lead system with the Mason-Likar modification. For analysis, the chest lead 5 (V5) was chosen. The resting ECG was measured from a healthy young male in relaxed conditions by using a NeuroScan system (Compumedics Limited, Tex, USA) with SynAmps ${ }^{2}$ amplifier. The sampling rate of the ECG signal was $1000 \mathrm{~Hz}$.

The exercise ECG recordings were performed by using a Cardiovit CS-200 ergospirometery system (Schiller AG) with Ergoline Ergoselect $200 \mathrm{~K}$ bicycle ergometer. The sampling rate of the ECG in the exercise recordings was $500 \mathrm{~Hz}$. Five healthy male subjects participated in the test (aged 27 to 33). In the stepwise test procedure shown in Figure 1, the subject first lay supine for three minutes and then sat up on the bicycle for the next three minutes. After that, the subject started the actual exercise part in which the load of the bicycle increased with $40 \mathrm{~W}$ every three minutes. The starting load was $40 \mathrm{~W}$ and the subject continued exercise until exhaustion. After the subject indicated that he could not go on anymore, the exercise test was stopped and a 10-minute recovery period was measured.

\subsection{Traditional RT interval measures}

Three different RT interval measurement methods are considered here, one $\mathrm{RT}_{\text {apex }}$ and two $\mathrm{RT}_{\text {end }}$ measures. First of all, it should be noted that especially the $\mathrm{RT}_{\text {end }}$ measures are very sensitive to ECG baseline drifts, and thus these lowfrequency trend components should be removed before analysis. Here, a 5th-order Butterworth highpass filter with cutoff frequency at $1 \mathrm{~Hz}$ was applied to remove the ECG baseline drifts. Secondly, all measures presume R-wave apex detection which is accomplished by using a QRS detection algorithm similar to the one presented in [15]. Once the R-wave apex is fixed, the T-wave apex or offset is searched from a window

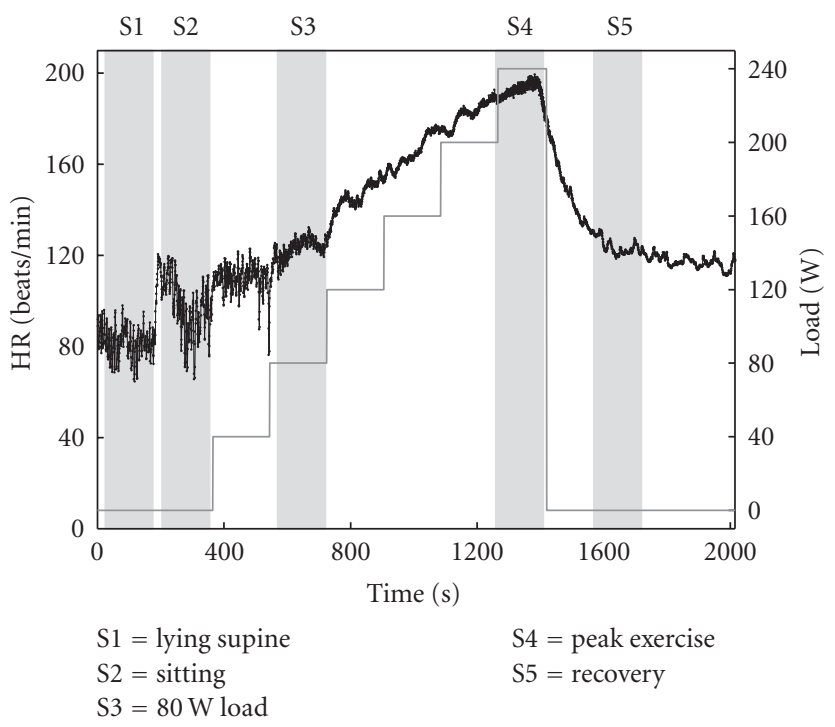

FIgure 1: The exercise test protocol for subject 1 showing the heart rate and bicycle load as functions of time. The samples selected for analysis $\mathrm{S} 1, \mathrm{~S} 2, \ldots, \mathrm{S} 5$ are indicated on top.

whose onset and offset (relative to the R-wave apex) are given as

$$
\begin{gathered}
{[100,500] \mathrm{ms} \quad \text { if } \mathrm{RR}_{\mathrm{av}}>700 \mathrm{~ms}} \\
{\left[100,0.7 \cdot \mathrm{RR}_{\mathrm{av}}\right] \mathrm{ms} \quad \text { if } \mathrm{RR}_{\mathrm{av}}<700 \mathrm{~ms}}
\end{gathered}
$$

where $R_{a v}$ is the average RR interval within the whole analyzed ECG recording. Similar window definition was used, for example, in [7].

The first considered method measures the time difference between R- and T-wave apexes as shown on Figure 2(a). First, the maximum of a lowpass filtered ECG is searched from window specified in (1). As the lowpass filter, a 20millisecond moving average FIR filter (for sampling rate of $1000 \mathrm{~Hz}$, filter order is 20 , filter coefficients $b_{j}=1 / 20$ for all $j=1, \ldots, 20$, and cutoff frequency $\sim 22 \mathrm{~Hz}$ ) was applied. Then, to reduce the effect of noise, a parabola is fitted around the T-wave maximum within a 60-millisecond frame and the T-wave apex is fixed as the maximum of the fitted parabola. This RT interval measure is here denoted by $\mathrm{RT}_{\text {apex }}$.

The second considered method measures the time difference between R-wave apex and T-wave offset by using a threshold technique as shown on Figure 2(b). To fix the Twave offset, the T-wave is first lowpass filtered by using the same moving average filter as in $\mathrm{RT}_{\text {apex }}$ measure. The $\mathrm{T}$-wave offset is then fixed as the intercept of the lowpass filtered Twave downslope with the threshold level above the isoelectric line. The isoelectric line is obtained as the amplitude value corresponding to the highest peak in the ECG histogram and the threshold level is set to $15 \%$ of the corresponding $\mathrm{T}$ wave maximum. This RT interval measure is here denoted by $\mathrm{RT}_{\mathrm{end}}^{(t)}$, where $t$ indicates threshold.

The third considered RT interval measure utilizes a line fit in T-wave offset determination as shown on Figure 2(c). The line fit is obtained as the steepest tangent of the lowpass 


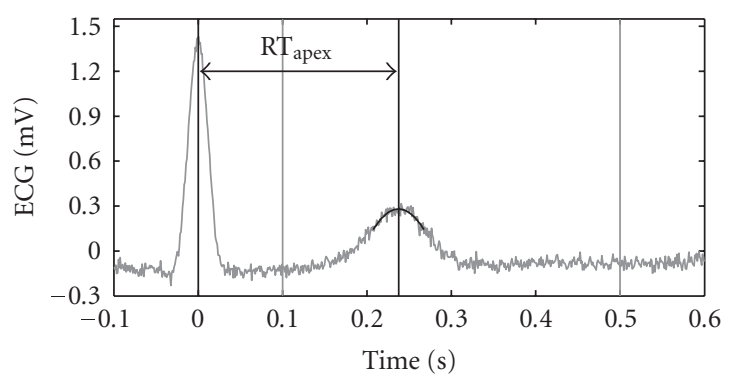

(a)

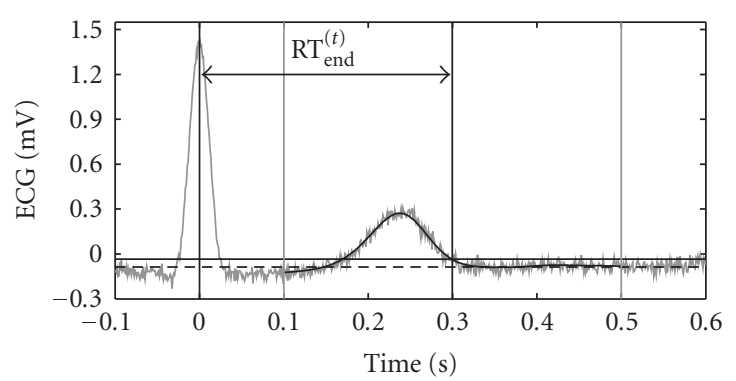

(b)

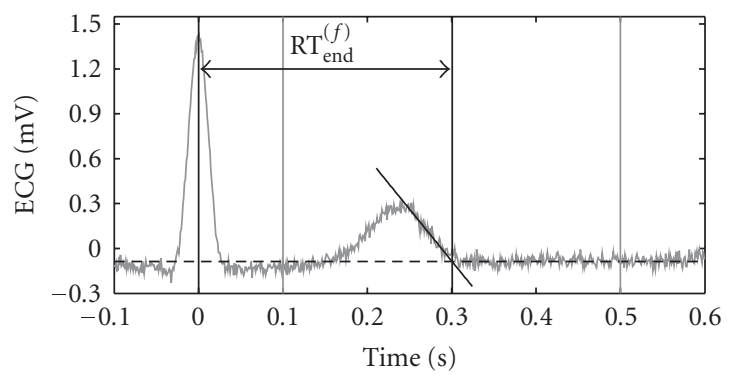

(c)

FIGURE 2: The three RT interval measurement methods considered: (a) $\mathrm{RT}_{\text {apex }}$, (b) $\mathrm{RT}_{\text {end }}^{(t)}$, and (c) $\mathrm{RT}_{\text {end }}^{(f)}$. The dashed line on the two bottommost axes indicates the isoelectric line.

filtered T-wave downslope (the same moving average filter as above). The T-wave offset is then fixed as the intercept of this tangent with the isoelectric line, where the isoelectric line is obtained as above. This RT interval measure is here denoted by $\mathrm{RT}_{\text {end }}^{(f)}$, where $f$ indicates fitting.

\subsection{Principal component regression approach}

In the principal component regression, the vector containing the measured signal is presented as a weighted sum of orthogonal basis vectors. The basis vectors are selected to be the eigenvectors of either the data covariance or correlation matrix. The central idea in PCR is to reduce the dimensionality of the data set, while retaining as much as possible of the variance in the original data [16].

In the PCR-based approach, the ECG measurement is first divided into adequate epochs such that each epoch includes a single T-wave. The T-wave epochs are extracted by applying the window specified in (1) for each heart-beat
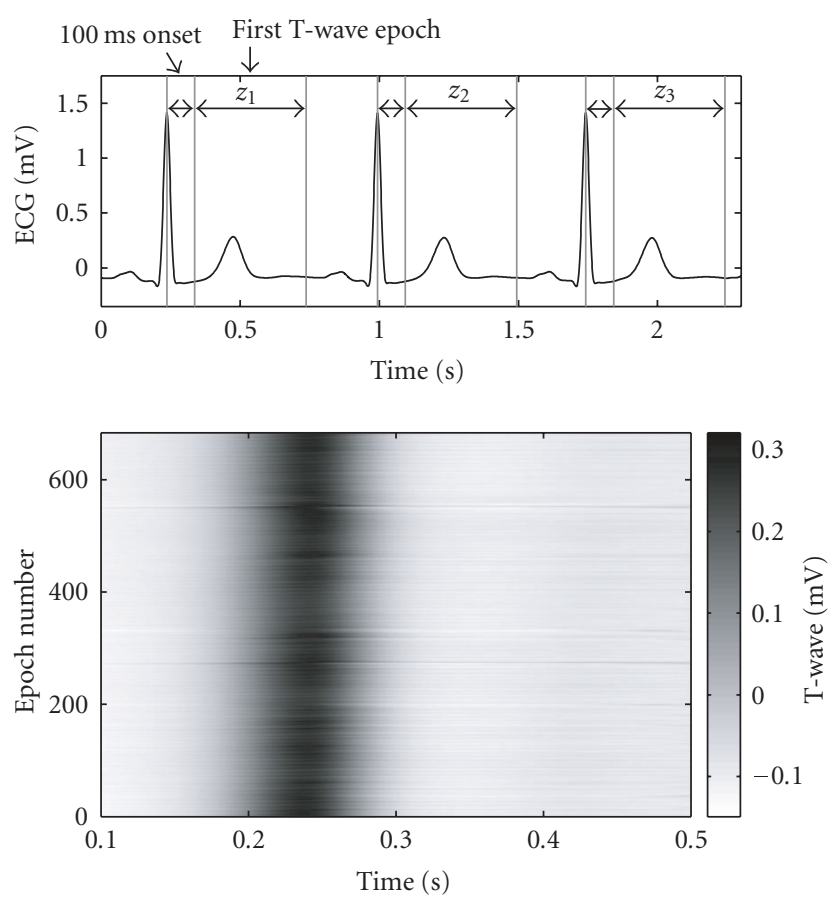

Figure 3: Extraction of T-wave epochs from the ECG recording.

period as shown in Figure 3. Note that the average RR interval $R_{a v}$ in (1) is calculated over the whole analyzed ECG recording, and thus the length of the extracted $\mathrm{T}$-wave epochs is constant. Let us denote such $j$ th epoch with a length $N$ column vector

$$
z_{j}=\left(\begin{array}{c}
z_{j}(1) \\
\vdots \\
z_{j}(N)
\end{array}\right) .
$$

As an observation model, we use the additive noise model

$$
z_{j}=s_{j}+e_{j},
$$

where $s_{j}$ is the noiseless ECG signal corresponding to $j$ th epoch and $e_{j}$ is the additive measurement noise. The measurement noise is assumed to be a stationary zero-mean process. If we have $M$ T-waves within the ECG recording, the signals $s_{j}$ will span a vector space $\&$ which will be at most of $\min \{M, N\}$ dimensions. In the case that the $\mathrm{T}$-wave epochs are rather similar, the dimension of this vector space will be $K \leq \min \{M, N\}$ and epochs $s_{j}$ can be well approximated with some lower-dimensional subspace of $\&$. Thus, each epoch can be expressed as a linear combination

$$
z_{j}=H_{s} \theta_{j}+e_{j}
$$

where $H_{s}=\left(\psi_{1}, \psi_{2}, \ldots, \psi_{K}\right)$ is an $N \times K$ matrix of basis vectors which span the $K$-dimensional subspace of $\delta$ and $\theta_{j}$ is a $K \times 1$ column vector of weights related to $j$ th epoch. By defining an $N \times M$ measurement matrix $z=\left(z_{1}, z_{2}, \ldots, z_{M}\right)$, the observation model (4) can be written in the form

$$
z=H_{s} \theta+e,
$$


where $\theta=\left(\theta_{1}, \theta_{2}, \ldots, \theta_{M}\right)$ is a $K \times M$ matrix of weights and $e=\left(e_{1}, e_{2}, \ldots, e_{M}\right)$ is an $N \times M$ matrix of error terms.

The critical point in the use of model (5) is the selection of the basis vectors $\psi_{k}$. A variety of ways to select these basis vectors exist, but here a special case, that is, principal component regression, is considered. In PCR, the basis vectors are selected to be the eigenvectors $v_{k}$ of either the data covariance or correlation matrix. Here the correlation matrix which can be estimated as

$$
R=\frac{1}{M} z z^{T}
$$

is utilized. The eigenvectors and the corresponding eigenvalues can be solved from the eigendecomposition. The eigenvectors of the correlation matrix are orthonormal, and therefore, the ordinary least-squares solution for the parameters $\theta$ becomes

$$
\hat{\theta}_{\mathrm{PC}}=H_{s}^{T} z
$$

and the T-wave estimates could be computed from

$$
\hat{z}_{\mathrm{PC}}=H_{s} \hat{\theta}_{\mathrm{PC}} \text {. }
$$

Quantitatively, the first basis vector is the best meansquare fit of a single waveform to the entire set of epochs. Thus, the first eigenvector is similar to the mean of the epochs and the corresponding parameter estimates or principal components (PCs) $\hat{\theta}_{j}(1)$ reveal the contribution of the first eigenvector to each epoch $(j=1,2, \ldots, M)$. The second eigenvector, on the other hand, covers mainly the variation in the $\mathrm{T}$-wave times and is expected to resemble the derivative of the T-wave. The model parameters corresponding to the second eigenvector, that is, the second PCs, are thus expected to reflect the variability of the time difference between R- and T-waves, that is, RT interval variability.

In conclusion, the second PCs are here taken as estimates for RT interval variability, and thus there is no need for Twave apex or offset detection. However, it should be noted that the PCs are in arbitrary units and do not yield absolute values for the RT intervals. If absolute RT interval values are desired, one should compute the T-wave estimates according to (8) and find the apexes or offsets of each estimate. In that case, the PCR approach could be seen just as a denoising procedure.

\subsection{Noise sensitivity of RT interval measures}

The most common approach for evaluating the noise sensitivity of an RT measurement method is to replicate a single noise-free cardiac cycle and add noise to hereby generated ECG. This leads to an ECG signal in which the "true" RT interval is constant and the noise sensitivity of the RT measurement method can be evaluated, for example, by determining the standard deviation of RT interval estimates for different noise levels. The proposed PCR-based method, however, assumes variability in RT interval, and thus cannot be evaluated this way. In fact, we are interested in the RT variability itself and want to evaluate the effect of noise on the RT variability estimates.
On way to accomplish this is to utilize some good quality ECG measurement which after preprocessing can be considered to be noise-free. The RT interval measures obtained from such noise-free ECG measurement can then be considered as the "true" RT intervals. To evaluate the noise sensitivity of different methods, Gaussian zero-mean noise of different levels can then be added to the noise-free ECG signal and different RT estimates may be recalculated for the noisy ECG. The observed changes in the RT variability series (compared to the "true" RT series) can be evaluated, for example, in frequency domain.

\section{RESULTS}

At first, we compared the PCR-based method with the three traditional RT interval measures by utilizing the resting ECG measurement. In order to remove measurement noise and to enable unambiguous detection of R- and T-waves, the ECG was bandpass filtered (passband $1-30 \mathrm{~Hz}$ ). The traditional RT interval measures when applied to this "noise-free" ECG may be considered to give accurate results against which the PCR method can be compared.

The T-wave epochs extracted from the noise-free ECG are shown in Figure 3. The correlation matrix for the epochs was calculated according to (6) and the first two eigenvectors of the correlation matrix are shown in Figure 4(a). The corresponding eigenvalues were $\lambda_{1}=0.9932$ and $\lambda_{2}=0.0041$. The first eigenvector clearly represents the mean of the ensemble and the second eigenvector is similar to the first derivative of the T-wave. As demonstrated in Figure 4(b), it is quite easy to see that in the superposition of the first two eigenvectors, the peak is moved according to the magnitude and sign of the second PC. For positive values of this component, the peak is moved to the right and for negative values to the left. Thus, the second PC can be used as a measure of RT interval variability, and even though, the second PC does not give absolute values for RT interval, it is here denoted as $\mathrm{RT}_{\mathrm{PC}}$.

The obtained RT interval variability series $\mathrm{RT}_{\mathrm{PC}}$ is compared with the traditional $\mathrm{RT}$ interval measures $\mathrm{RT}_{\mathrm{apex}}$, $\mathrm{RT}_{\text {end }}^{(t)}$, and $\mathrm{RT}_{\text {end }}^{(f)}$ in Figure 5. It is observed that the variation in the $\mathrm{RT}_{\mathrm{PC}}$ is very similar to the variations in the traditional RT measures. Even the deviations at about 200 and 400 seconds seem to be captured by the PCR method. The similarity of the $\mathrm{RT}_{\mathrm{PC}}$ series with the traditional RT series was further evaluated both in frequency and in time domain. In frequency domain, the power-spectrum estimates of different RT series were calculated by using Welch's periodogram method. Prior to spectrum estimation, each RT series was converted to evenly sampled series by using a $4 \mathrm{~Hz}$ cubic spline interpolation and the trend was removed by using a smoothness-priors-based method presented in [17].

The obtained spectrum estimates for different RT measures presented in Figure 5 seem to have similar shape. The percentual powers of low-frequency (LF, 0.04-0.15 Hz) and high-frequency (HF, $0.15-0.4 \mathrm{~Hz}$ ) bands, $\mathrm{LF} / \mathrm{HF}$ ratio, as well as the LF and HF peak frequencies were then calculated. The obtained results are presented in Table 1 . In time domain, the correlation coefficients between $\mathrm{RT}_{\mathrm{PC}}$ and the traditional 


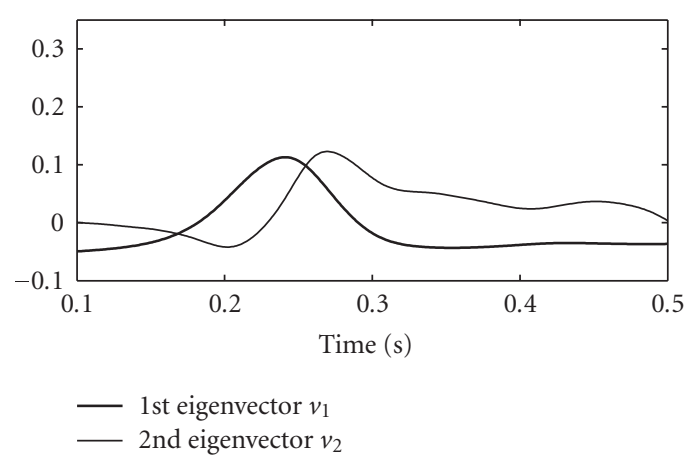

(a)

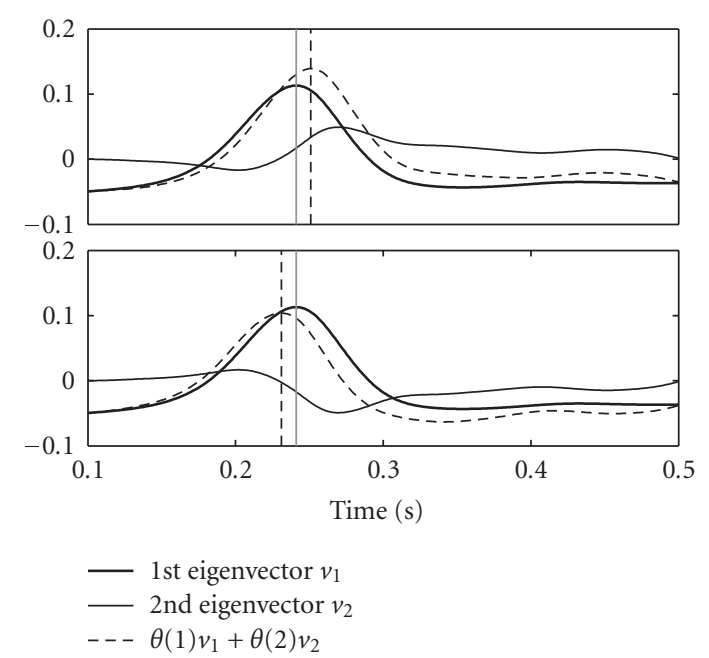

(b)

FIGURE 4: Demonstration of T-wave latency jitter modeling by the first two eigenvectors. (a) The first two eigenvectors of the T-wave epochs and (b) the superposition of these eigenvectors when the second PC is positive (top) or negative (bottom).

measures were calculated. These coefficients and the corresponding correlation plots are shown on the right-hand side of Figure 5. The obtained correlation coefficients are quite high considering that the corresponding coefficients between the traditional measures were not considerably higher as can be seen from Table 1 .

The noise sensitivity of the different RT variability estimates was then evaluated by adding Gaussian zero-mean noise to the noise-free ECG. The noise levels applied were such that the SNRs of the generated noisy ECG signals were $50,40,30,25,20,15,10$, and 5 decibels, see Figure 6 . For each noise level, the $\mathrm{RT}_{\text {apex }}, \mathrm{RT}_{\text {end }}^{(t)}, \mathrm{RT}_{\text {end }}^{(f)}$, and $\mathrm{RT}_{\mathrm{PC}}$ measures were reevaluated and the corresponding spectrum estimates were calculated as before. The distortion of the spectrum estimates for decreased SNRs was clearly observed especially for traditional RT measures.

This distortion was then quantified by generating a total of 1000 noisy ECG realizations for each noise level and by evaluating the relative LF and HF band powers for each realization and for each RT variability measure. The obtained results are presented in Figure 7, where the mean band powers and their SD intervals are presented for each RT measure as a function of SNR. The SNR $=\infty$ corresponds to the noisefree ECG signal.

Finally, the proposed method and the three traditional RT measures were applied to the exercise ECG measurements. Five samples were chosen for analysis from each measurement according to Figure 1 . These stages were S1 = lying supine, $\mathrm{S} 2=$ sitting, $\mathrm{S} 3=80 \mathrm{~W}$ load, $\mathrm{S} 4=$ peak exercise, and S5 = recovery stage. Each analyzed sample was 150 seconds of length. $\mathrm{RT}_{\text {apex }}, \mathrm{RT}_{\text {end }}^{(t)}, \mathrm{RT}_{\text {end }}^{(f)}$, and $\mathrm{RT}_{\mathrm{PC}}$ measures as well as RR intervals were then extracted from every sample. The obtained time series for one subject are presented in Figure 8(a). This particular subject had prominent T-wave throughout the measurement, and practically all the RT measures were obtained without significant problems. However, in two of the subjects having weaker T-waves, the traditional RT measures showed significant errors especially near peak exercise.

Note that each RT measure and RR series in Figure 8(a) are presented in the same scale for all stages, and thus for example, the decrease in RR variability during exercise is evident. For traditional RT measures, on the other hand, the variability seems to increase during exercise which is, however, probably mainly due to the effect of noise. For the proposed method, the variability levels between different stages are not comparable because the PCR method is applied separately to each stage, and for example, the eigenvectors are different in each stage.

Figure 8(b) presents the detrended RR and RT series, where the trend was removed by using the smoothness priors method. Note that each detrended series is presented in a minmax scale to permit the visualization of similarities/differences among series, and thus there are no scales for RR or RT interval durations.

The power-spectrum estimates were then calculated for each detrended series and each stage by using Welch's periodogram method as before. The obtained spectrum estimates are presented in Figure 8(c), where each spectrum has been divided into three frequency bands: low frequency (LF, $0.04-0.15 \mathrm{~Hz}$ ), high frequency (HF, 0.15-0.4 Hz), and very high frequency (VHF, $0.4-1 \mathrm{~Hz}$ ) according to [18]. In addition, the mean respiratory frequencies observed from the spirometer measurements for each stage are marked with dashed lines. The observed respiratory frequencies were 0.34 , $0.31,0.31,0.55$, and $0.49 \mathrm{~Hz}$ for stages S1, S2, S3, S4, and S5, respectively. It should, however, be noted that within most of the stages, the respiratory frequency varied significantly around its mean value.

Note that each spectrum estimate is displayed in different scales to enable the comparison of spectral shapes, and thus there is no power scale in Figure 8(c). The spectra of different RT variability estimates have clearly similar characteristics which are partly congruent with the RR spectra. These spectral properties are further compared in Figure 9, where relative LF, HF, and VHF band powers for RR interval series 

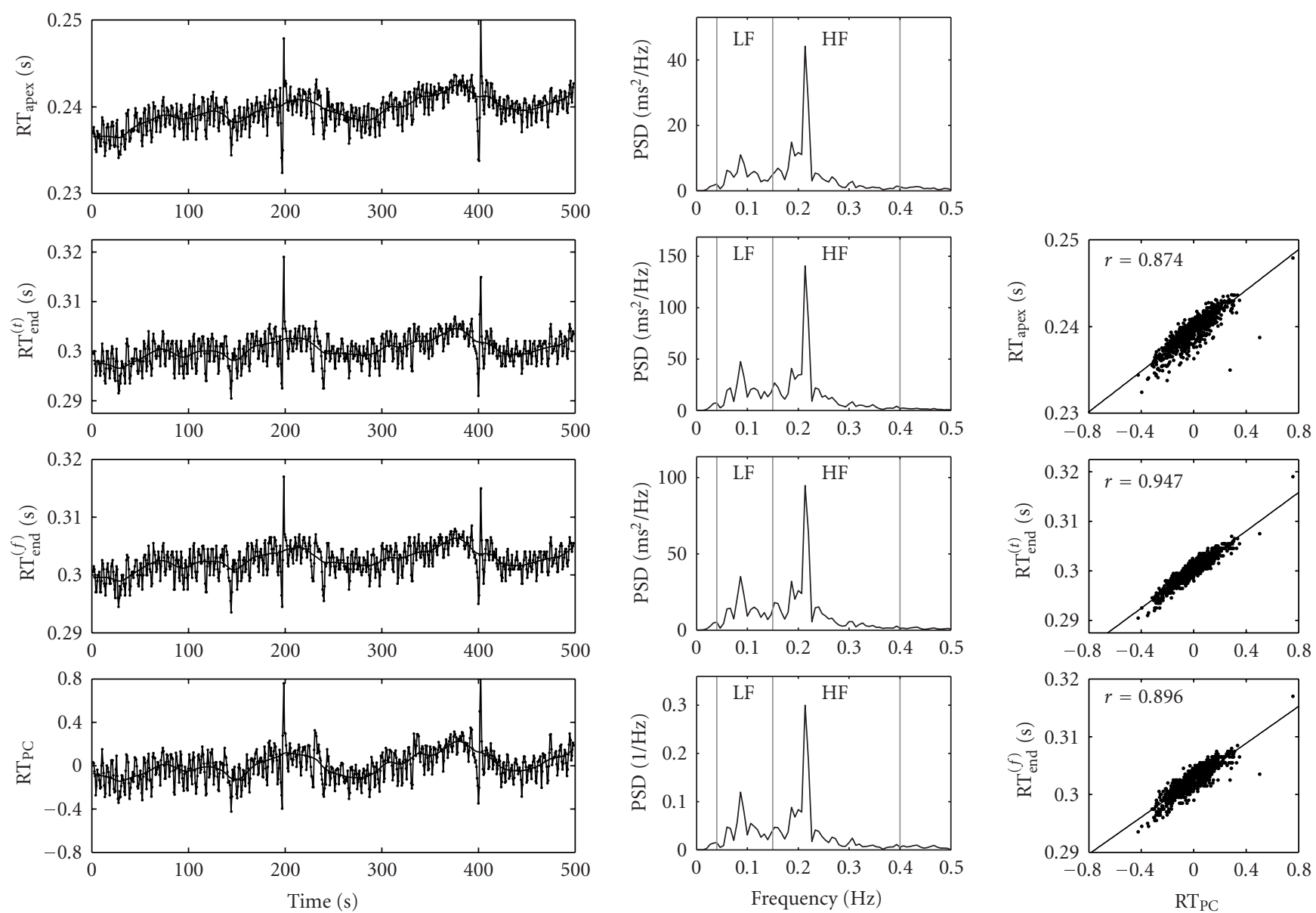

(a)

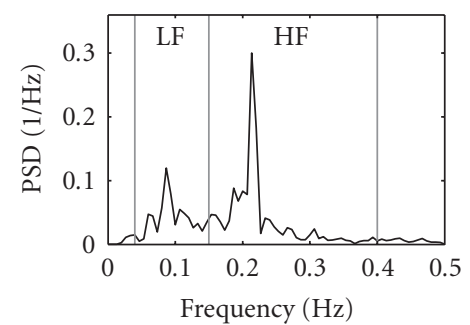

(b)

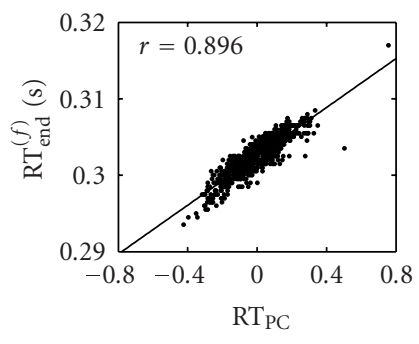

(c)

FIGURE 5: Comparison of the RT interval variability series $\mathrm{RT}_{\mathrm{PC}}$ (obtained by the PCR-based method) with traditional RT interval measures $\mathrm{RT}_{\text {apex }}, \mathrm{RT}_{\text {end }}^{(t)}$, and $\mathrm{RT}_{\text {end }}^{(f)}$. (a) The different RT measures and the estimated trend, (b) corresponding spectrum estimates, and (c) correlation plots.

TABLE 1: Spectral variables and correlation coefficients of different RT interval measures presented in Figure 5.

\begin{tabular}{lcccc}
\hline & $\mathrm{RT}_{\text {apex }}$ & $\mathrm{RT}_{\text {end }}^{(t)}$ & $\mathrm{RT}_{\text {end }}^{(f)}$ & $\mathrm{RT}_{\mathrm{PC}}$ \\
\hline Spectral variables & & & & \\
LF power (\%) & 27.9 & 31.6 & 31.4 & 32.9 \\
HF power (\%) & 70.6 & 66.8 & 67.0 & 65.4 \\
LF/HF ratio & 0.395 & 0.474 & 0.469 & 0.502 \\
LF peak (Hz) & 0.087 & 0.087 & 0.087 & 0.087 \\
HF peak (Hz) & 0.213 & 0.213 & 0.214 & 0.213 \\
\hline Correlation coefficients, $r$ & & & & \\
$\mathrm{RT}_{\text {apex }}$ & - & 0.892 & 0.918 & 0.874 \\
$\mathrm{RT}_{\text {end }}^{(t)}$ & - & - & 0.966 & 0.947 \\
$\mathrm{RT}_{\text {end }}^{(f)}$ & - & - & - & 0.896 \\
\hline
\end{tabular}

and for the different RT measures are presented for all five subjects as a function of the stage.

\section{DISCUSSION}

Ventricular repolarization duration variability, which is typically assessed by examining the variability within the RT interval, is a potential tool in cardiovascular research. Various algorithms for estimating RT interval from ECG have been applied, see, for example, [3, 5-10, 13, 19]. Considering the rather low spontaneous variability within the RT interval, the need for high precision in the measurement of this interval is obvious. The detection of the rather smooth T-wave can, however, be problematic especially in low SNR conditions. In this paper, we have proposed a new PCR-based method for estimating the RT interval variability. The main benefit of the proposed method is that it does not necessitate T-wave detection.

The proposed method was compared with traditional $\mathrm{RT}_{\text {apex }}$ and $\mathrm{RT}_{\text {end }}$ measures by using a good-quality (practically noise-free) ECG measurement and the proposed method was observed to be highly congruent with the traditional RT measures as can be seen from Figure 5 and Table 1. Both the spectral characteristics and time-domain 

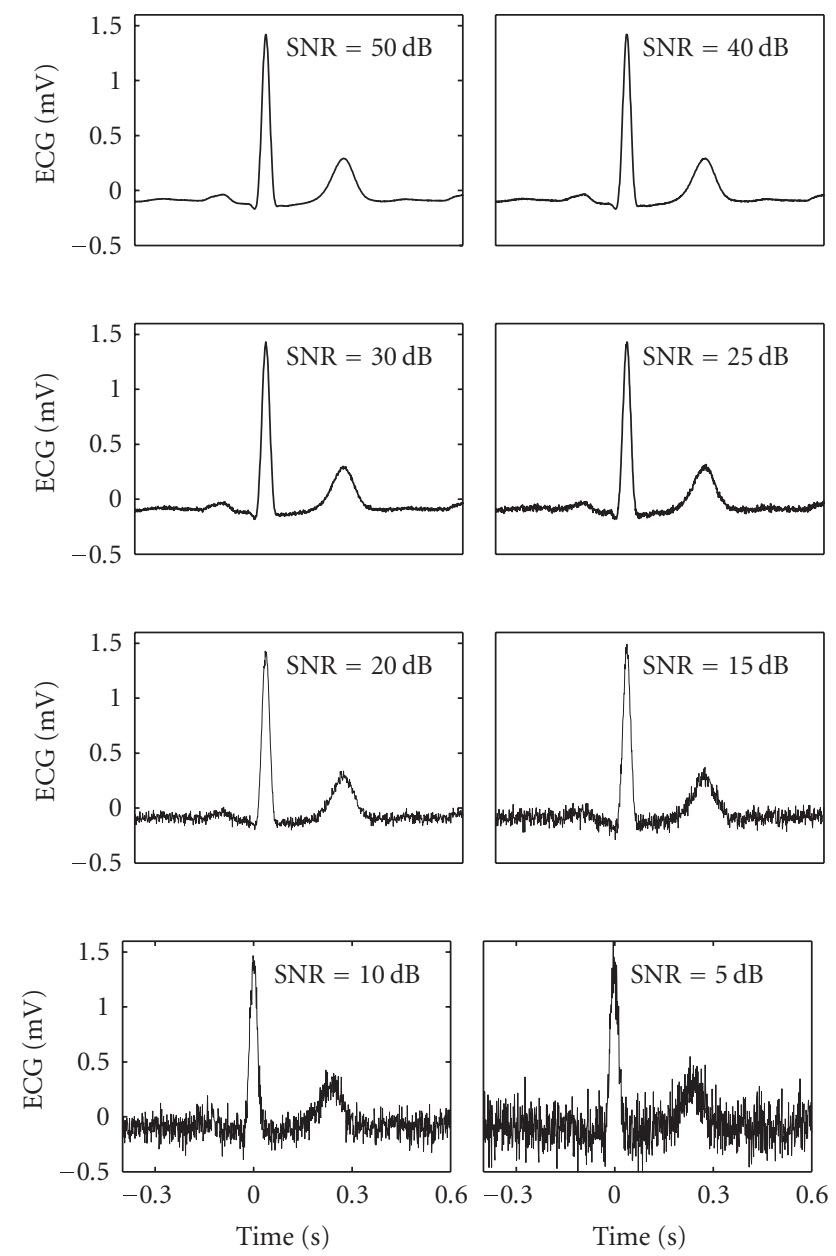

FIGURE 6: Samples of the generated noisy ECG signals with different SNRs.

correlations of the estimated RT variability series were compared. These results indicate that the proposed PCR-based method estimates RT variability correctly.

In the proposed method, RT variability is modeled by the second eigenvector of data correlation matrix. The first few eigenvectors tend to describe the main features of the data set, which in this case include T-wave shape and position, and thus the method is expected to be quite robust to noise. The noise sensitivity of the proposed method was tested by generating noisy ECG signals with SNRs between 50 and $5 \mathrm{~dB}$. For each SNR, the spectrum estimates of the estimated RT variability series were calculated and LF and HF band powers were evaluated. The proposed method was clearly less sensitive to noise when compared to the traditional RT measures as can be seen from Figure 7. When comparing the traditional methods, the $\mathrm{RT}_{\text {apex }}$ measure was observed to be the most precise in the presence of noise, which is in agreement with previous studies $[8,9,13]$.

It should be noted that in the PCR method, the noisy ECG was not preprocessed in any way, and thus it can be concluded that the method is very robust to noise, at
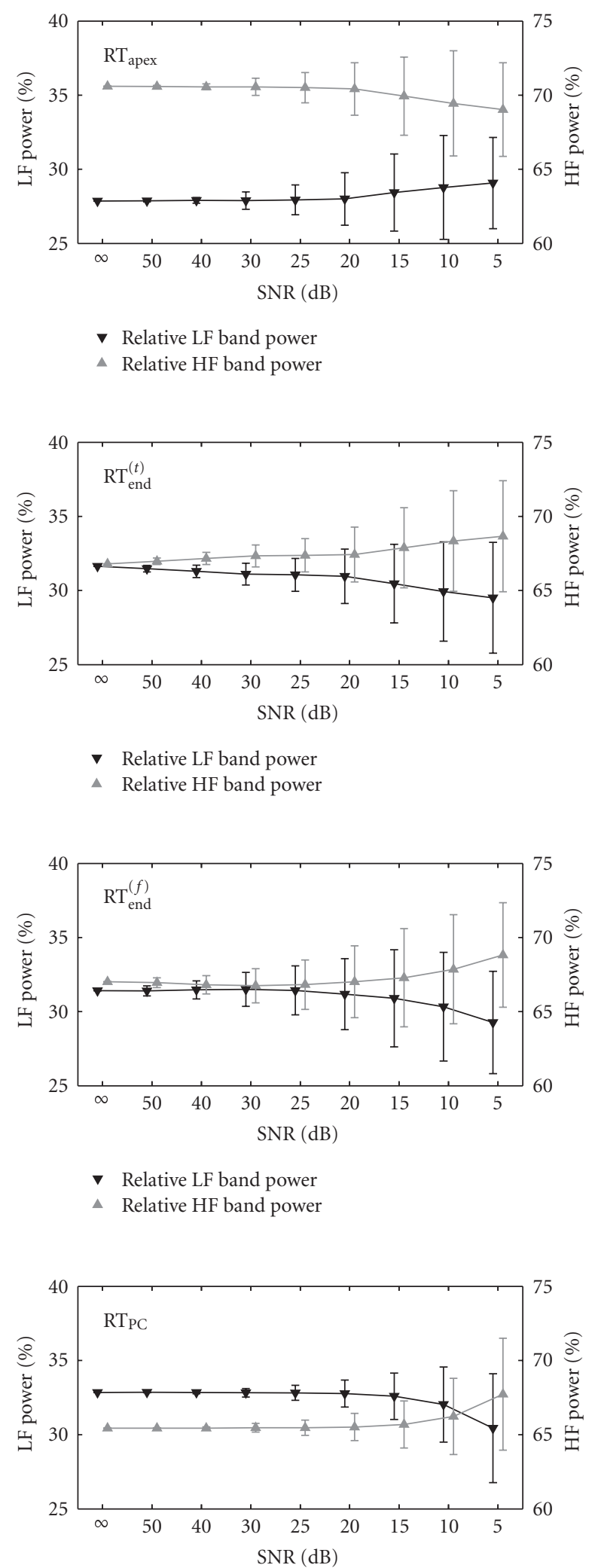

V Relative LF band power

$\Delta$ Relative HF band power

FIGURE 7: The noise sensitivity of the different RT variability estimates. Relative LF $(\boldsymbol{\nabla})$ and HF $(\Delta)$ band powers with SD intervals for $\mathrm{RT}_{\text {apex }}, \mathrm{RT}_{\text {end }}^{(t)}, \mathrm{RT}_{\text {end }}^{(f)}$, and $\mathrm{RT}_{\mathrm{PC}}$ as a function of $\mathrm{SNR}$. 

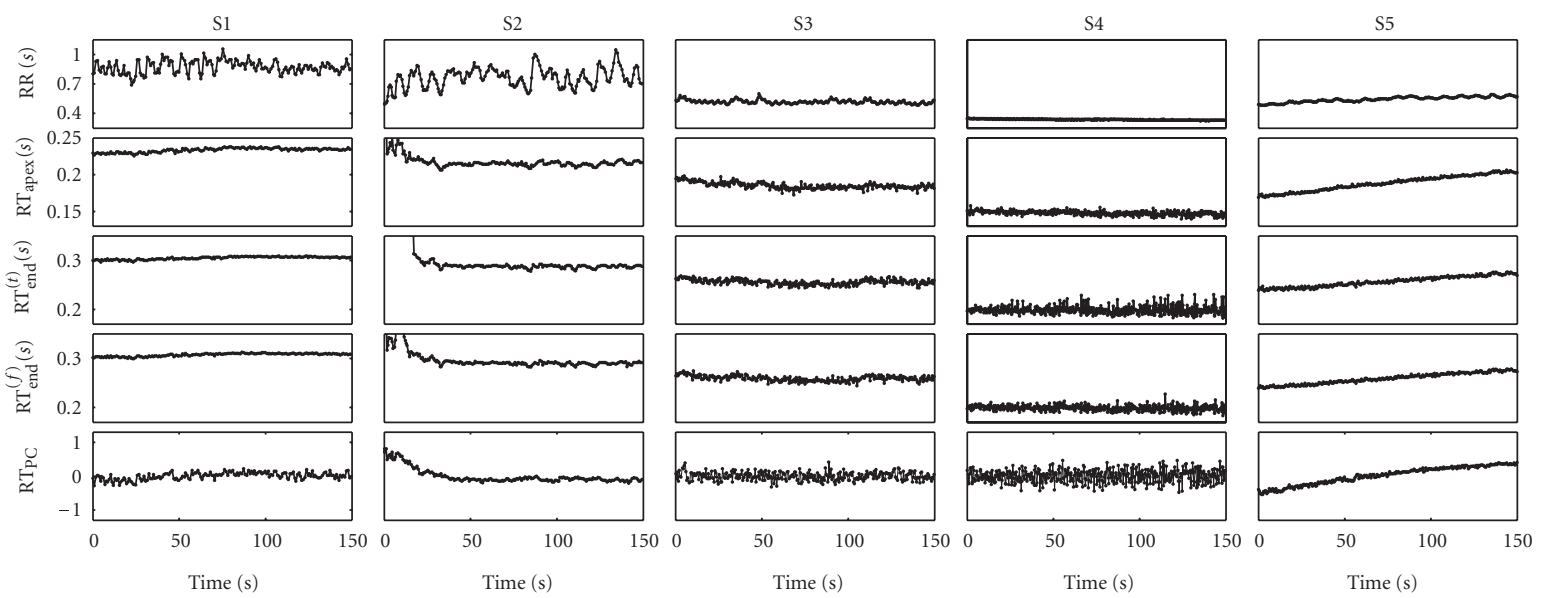

(a)
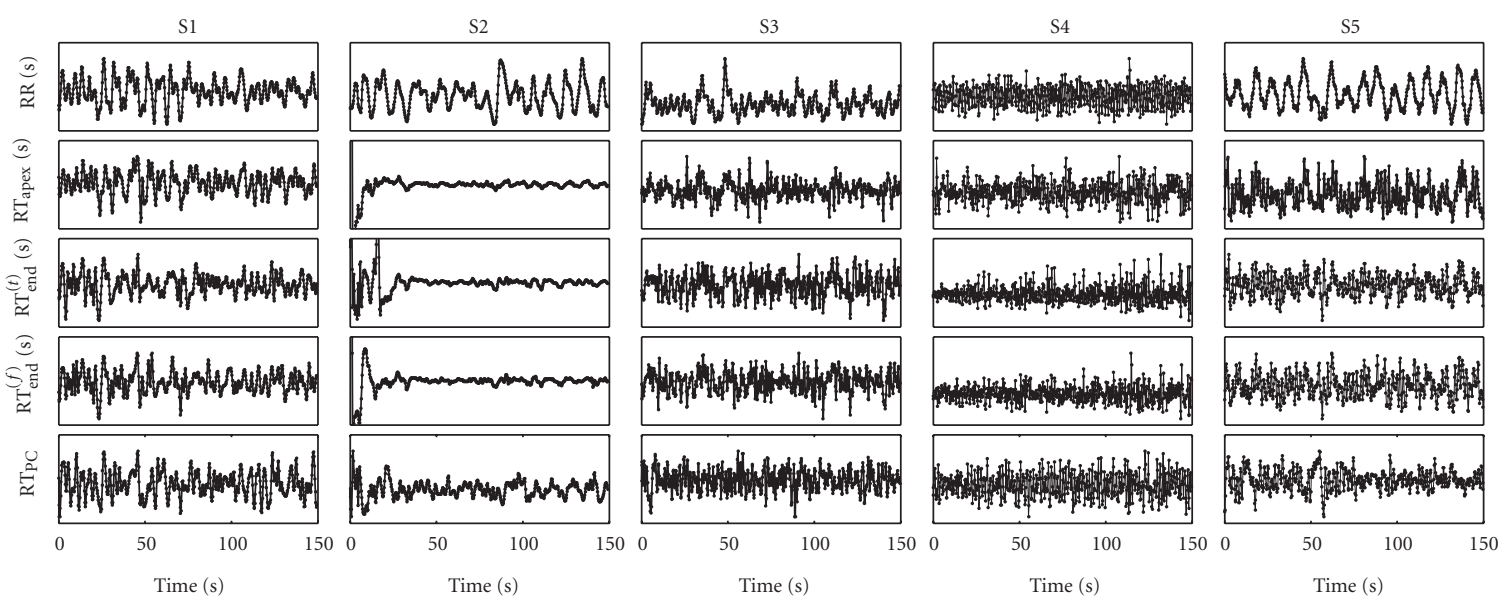

(b)
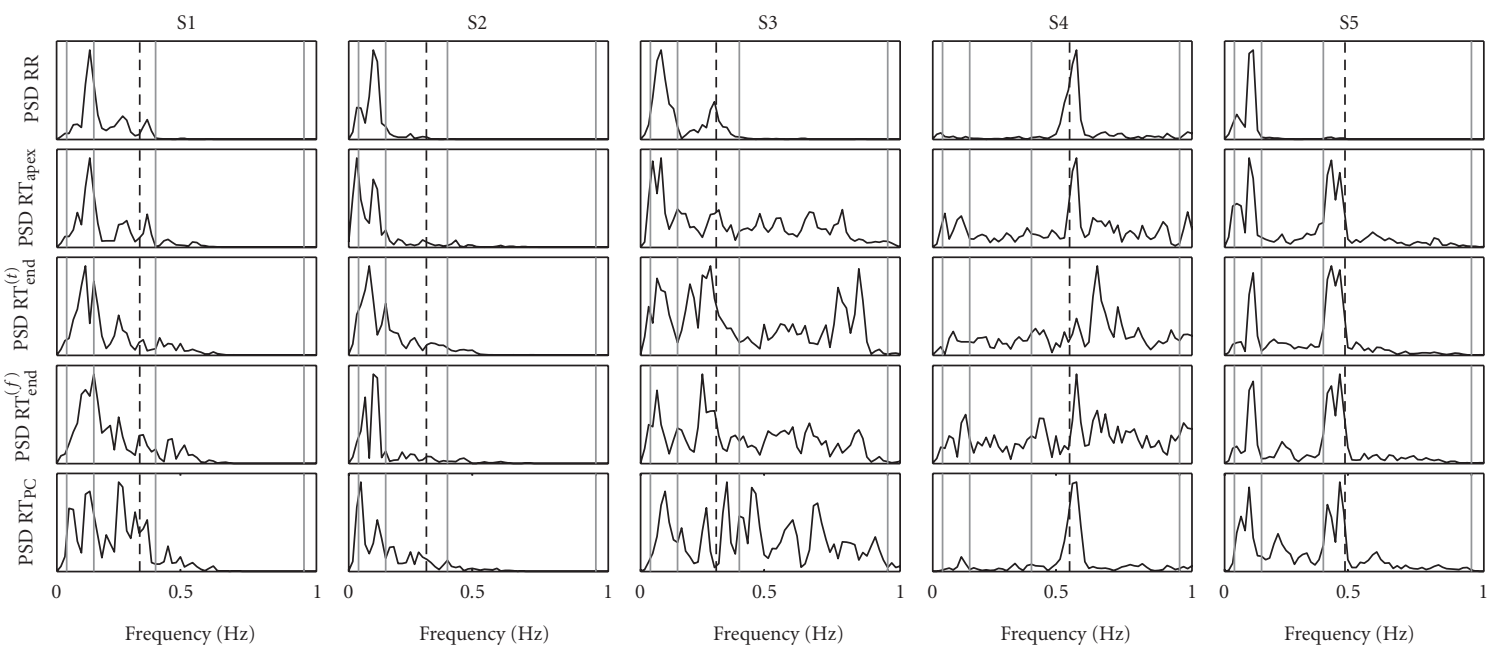

(c)

FIGURE 8: Exercise ECG measurement of one subject. (a) RR interval, $\mathrm{RT}_{\text {apex }}, \mathrm{RT}_{\text {end }}^{(t)}, \mathrm{RT}_{\text {end }}^{(f)}$, and $\mathrm{RT}_{\mathrm{PC}}$ series and (b) the corresponding detrended series for stages S1, S2, .., S5. (c) Corresponding spectrum estimates with gray lines indicating the LF, HF, and VHF bands and the dashed line indicating the mean observed respiratory frequency. 

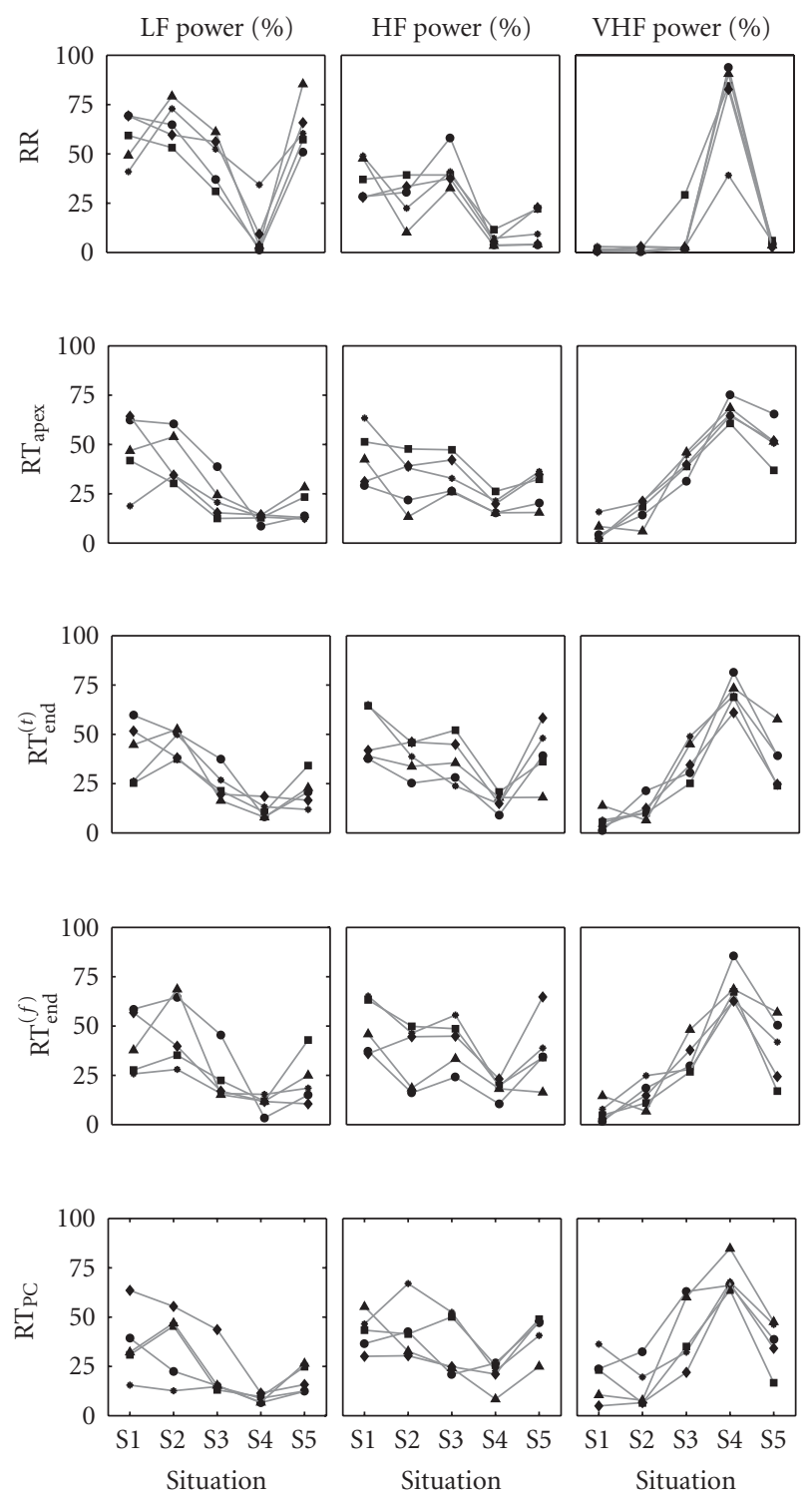

Figure 9: Exercise ECG measurement results. Relative LF, HF, and VHF band powers for RR interval, $\mathrm{RT}_{\mathrm{apex}}, \mathrm{RT}_{\mathrm{end}}^{(t)}, \mathrm{RT}_{\text {end }}^{(f)}$, and $\mathrm{RT}_{\mathrm{PC}}$ series for stages $\mathrm{S} 1, \mathrm{~S} 2, \ldots, \mathrm{S} 5$. Each line represents results of one subject.

least to Gaussian noise. Baseline oscillations, on the other hand, would most probably cause significant distortion to the method and should, thus, be removed before the PCR analysis. Another issue which can cause significant distortion and should be taken care of before analysis is if the T-wave morphology changes remarkably within the measurement. However, these limitations have more or less effect also on the traditional RT measures applied in this paper.

Lastly, the proposed method was applied to a set of exercise ECG measurements in which high noise levels are observed especially near the peak exercise. Five samples were chosen for analysis according to Figure 1 and the estimated RT variability series along with the corresponding RR inter- val series for one subject were presented in Figure 8. In RR variability, an increase in the relative VHF power is observed in peak exercise, which is in agreement with previous findings $[18,20]$. The RT variability is observed to have similar spectral characteristics as RR variability with two major differences. First of all, during stage S3, RT variability is characterized by a more pronounced VHF component than RR variability. Secondly, in all RT variability estimates, the relative power of the VHF component seems to remain high also in the recovery stage unlike in RR variability as can be seen from Figure 9.

\section{CONCLUSIONS}

In conclusion, the proposed method is a potential approach for studying RT interval variability. The method is very robust to noise and gives results which are congruent with traditional RT variability measures. The method is also rather simple to apply, requiring only the detection of the strong ECG R-wave. Probably, the main drawback of the method is that it does not directly give absolute values for RT interval. The absolute values could, however, be estimated by evaluating the relationship between the second principal components and the corresponding T-wave positions (see Figure 4), or simply by evaluating the T-wave apexes or offsets from the T-wave estimates obtained from (8).

\section{REFERENCES}

[1] M. Merri, A. J. Moss, J. Benhorin, E. H. Locati, M. Alberti, and F. Badilini, "Relation between ventricular repolarization duration and cardiac cycle length during 24-hour Holter recordings: findings in normal patients and patients with long QT syndrome," Circulation, vol. 85, no. 5, pp. 1816-1821, 1992.

[2] W. Zareba and A. B. de Luna, "QT dynamics and variability," The Annals of Noninvasive Electrocardiology, vol. 10, no. 2, pp. 256-262, 2005.

[3] R. D. Berger, "QT variability," Journal of Electrocardiology, vol. 36, supplement 1, pp. 83-87, 2003.

[4] R. Negoescu, S. Dinca-Panattescu, V. Filcescu, D. Ionescu, and S. Wolf, "Mental stress enhances the sympathetic fraction of QT variability in an RR-independent way," Integrative Physiological and Behavioral Science, vol. 32, no. 3, pp. 220-227, 1997.

[5] M. Merri, M. Alberti, and A. J. Moss, "Dynamic analysis of ventricular repolarization duration from 24-hour Holter recordings," IEEE Transactions on Biomedical Engineering, vol. 40, no. 12, pp. 1219-1225, 1993.

[6] G. Nollo, G. Speranza, R. Grasso, R. Bonamini, L. Mangiardi, and R. Antolini, "Spontaneous beat-to-beat variability of the ventricular repolarization duration," Journal of Electrocardiology, vol. 25, no. 1, pp. 9-17, 1992.

[7] P. Laguna, N. V. Thakor, P. Caminal, et al., "New algorithm for QT interval analysis in 24-hour Holter ECG: performance and applications," Medical and Biological Engineering and Computing, vol. 28, no. 1, pp. 67-73, 1990.

[8] A. Porta, G. Baselli, F. Lombardi, et al., "Performance assessment of standard algorithms for dynamic R-T interval measurement: comparison between R- $T_{\text {apex }}$ and R- $T_{\text {end }}$ approach," Medical and Biological Engineering and Computing, vol. 36, no. 1, pp. 35-42, 1998. 
[9] P. E. Tikkanen, L. C. Sellin, H. O. Kinnunen, and H. V. Huikuri, "Using simulated noise to define optimal QT intervals for computer analysis of ambulatory ECG," Medical Engineering and Physics, vol. 21, no. 1, pp. 15-25, 1999.

[10] P. P. Davey, " $Q T$ interval measurement: $Q$ to $T_{\text {apex }}$ or $Q$ to $T_{\text {end }}$ ?" Journal of Internal Medicine, vol. 246, no. 2, pp. 145149, 1999.

[11] I. Savelieva, G. Yi, X.-H. Guo, K. Hnatkova, and M. Malik, "Agreement and reproducibility of automatic versus manual measurement of QT interval and QT dispersion," The American Journal of Cardiology, vol. 81, no. 4, pp. 471-477, 1998.

[12] R. H. Ireland, R. T. C. E. Robinson, S. R. Heller, J. L.B. Marques, and N. D. Harris, "Measurement of high resolution ECG QT interval during controlled euglycaemia and hypoglycaemia," Physiological Measurement, vol. 21, no. 2, pp. 295303, 2000.

[13] G. Speranza, G. Nollo, F. Ravelli, and R. Antolini, "Beat-tobeat measurement and analysis of the R-T interval in $24 \mathrm{~h}$ ECG Holter recordings," Medical and Biological Engineering and Computing, vol. 31, no. 5, pp. 487-494, 1993.

[14] G.-X. Yan and C. Antzelevitch, "Cellular basis for the normal T wave and the electrocardiographic manifestations of the longQT syndrome," Circulation, vol. 98, no. 18, pp. 1928-1936, 1998.

[15] J. Pan and W. J. Tompkins, "A real-time QRS detection algorithm," IEEE Transactions on Biomedical Engineering, vol. 32, no. 3, pp. 230-236, 1985.

[16] I. T. Jolliffe, Principal Component Analysis, Springer, New York, NY, USA, 1986.

[17] M. P. Tarvainen, P. O. Ranta-Aho, and P. A. Karjalainen, "An advanced detrending method with application to HRV analysis," IEEE Transactions on Biomedical Engineering, vol. 49, no. 2, pp. 172-175, 2002.

[18] R. Bailón, J. Mateo, S. Olmos, et al., "Coronary artery disease diagnosis based on exercise electrocardiogram indexes from repolarisation, depolarisation and heart rate variability," Medical and Biological Engineering and Computing, vol. 41, no. 5, pp. 561-571, 2003.

[19] M. Merri, J. Benhorin, M. Alberti, E. Locati, and A. J. Moss, "Electrocardiographic quantitation of ventricular repolarization," Circulation, vol. 80, no. 5, pp. 1301-1308, 1989.

[20] J. Mateo, P. Serrano, R. Bailón, et al., "Heart rate variability measurements during exercise test may improve the diagnosis of ischemic heart disease," in Proceedings of the 23rd Annual International Conference of the IEEE Engineering in Medicine and Biology Society (EMBS '01), vol. 1, pp. 503-506, Istanbul, Turkey, October 2001.

Mika P. Tarvainen received the M.S. degree in 1999 and the Ph.D. degree in 2004 from the University of Kuopio, Finland. His Ph.D. research was concerned with estimation methods for nonstationary biosignals. Since 1999, he has been working in the Department of Physics, University of Kuopio as a Researcher. He is currently a Senior Researcher and a Lecturer of the Signal Analysis Course in the Department of Physics. His

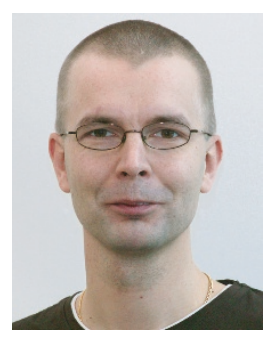
current research area includes biomedical signal analysis methods and their applications. In methodological research, he has focused on time series and spectral estimation methods, time-varying estimation methods, and nonlinear techniques.
Tomi Laitinen received the M.D. degree in 1991 and the Ph.D. degree in 2000 from the University of Kuopio, Finland. His Ph.D. research was concerned with physiological correlates of the cardiovascular variability. Since 2004, he has been a University Docent (Adjunct Professor) in the Department of Clinical Physiology and Nuclear Medicine in University of Kuopio. He is currently a Clinical Lecturer in University of Kuopio

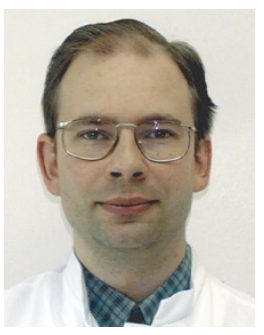
and Consultant in the Department of Clinical Physiology and $\mathrm{Nu}-$ clear Medicine in Kuopio University Hospital. His current research is focused on physiology and pathophysiology of cardiovascular regulation and vascular function.

Tiina Lyyra-Laitinen received the M.S. degree in 1991, the Ph.D. degree in 1998, and degree of Hospital Physicist from the University of Kuopio, Finland. Her Ph.D. research was concerned with arthroscopic measurement of knee-joint cartilage stiffness. She is currently a Hospital Physicist in the Department of Clinical Physiology and Nuclear Medicine, Kuopio University Hospital. Her current research activities include cardiovascular biomechanics and signal analysis.

Juha-Pekka Niskanen received the M.S. degree in medical physics from University of Kuopio, Kuopio, Finland, in 2006. He is currently working in University of Kuopio, Department of Physics as a Researcher. His current research is focused on the applications of biomedical signal processing and functional magnetic resonance imaging.
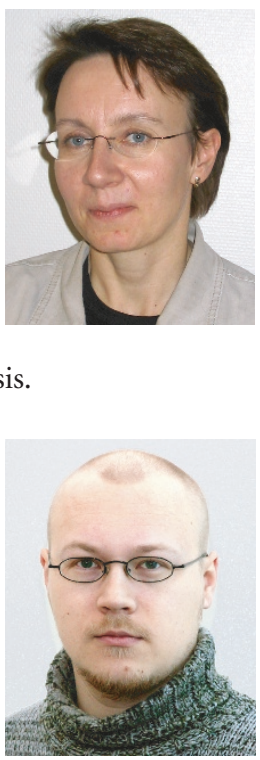

Pasi A. Karjalainen received the Ph.D. degree in 1997 from the University of Kuopio, Finland. Since 1988, he has been working in University of Kuopio as Researcher and in Kuopio University Hospital as Physicist. He is currently a Professor in the Department of Physics and he is leading the Research Group of Biomedical Signal Analysis and Medical Imaging. His research areas include biomedical signal analysis and medical imaging applications. Most of his work has been concerned with application of Bayesian and regularization methods to biomedical problems.

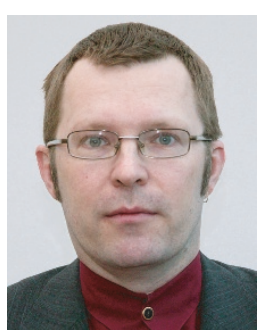

